\title{
On a uniqueness theorem of
} Sturm-Liouville equations with boundary conditions polynomially dependent on the spectral parameter

\author{
Yu Ping Wang ${ }^{1}$, Ko Ya Lien² and Chung Tsun Shieh ${ }^{2 *}$ (D)
}

\section{"Correspondence:}

ctshieh@mail.tku.edu.tw

${ }^{2}$ Department of Mathematics,

Tamkang University, New Taipei City, Taiwan

Full list of author information is

available at the end of the article

\begin{abstract}
Inverse nodal problems for Sturm-Liouville equations associated with boundary conditions polynomially dependent on the spectral parameter are studied. The authors show that a twin-dense subset $W_{B}([a, b])$ can uniquely determine the operator up to a constant translation of eigenparameter and potential, where $[a, b]$ is an arbitrary interval which contains the middle point of the domain of the operator and $B$ is a subset of $\mathbb{N}$ which satisfies some condition (see Theorem 4.2).
\end{abstract}

MSC: 34A55; 34B24; 47E05

Keywords: Inverse spectral problem; Inverse nodal problem; Spectral parameter; Potential; Weyl m-function

\section{Introduction}

The inverse problems of the differential operator $L:=L\left(q, U_{0}, U_{1}\right)$ :

$$
\left\{\begin{array}{l}
l u:=-u^{\prime \prime}+q(x) u=\lambda u, \quad x \in(0,1), \\
U_{0}(u):=R_{01}(\lambda) u^{\prime}(0, \lambda)+R_{00}(\lambda) u(0, \lambda)=0, \\
U_{1}(u):=R_{11}(\lambda) u^{\prime}(1, \lambda)+R_{10}(\lambda) u(1, \lambda)=0,
\end{array}\right.
$$

are considered, where $\lambda$ is called the spectral parameter, $q$ is a real-valued $L^{2}$-function on $(0,1)$ and

$$
R_{\xi k}(\lambda)=\sum_{l=0}^{r_{\xi k}} a_{\xi k l} \lambda^{r_{\xi k}-l}, \quad r_{\xi 1}=r_{\xi 0}=r_{\xi} \geq 0, a_{\xi 10}=1, \xi, k=0,1,
$$

are arbitrary polynomials of degree $r_{\xi}$ with real coefficients such that $R_{\xi 1}(\lambda)$ and $R_{\xi 0}(\lambda)$ have no common zeros for $\xi=0,1$. The inverse spectral problem for the Sturm-Liouville equation with boundary conditions dependent on the spectral parameter was studied in [1-7] respectively. In particular, Freiling and Yurko [4] studied three inverse spectral problems for $L$ and showed that this operator $L$ can be recovered either from the Weyl function,

(c) The Author(s) 2018. This article is distributed under the terms of the Creative Commons Attribution 4.0 International License (http://creativecommons.org/licenses/by/4.0/), which permits unrestricted use, distribution, and reproduction in any medium, provided you give appropriate credit to the original author(s) and the source, provide a link to the Creative Commons license, and indicate if changes were made. 
or from discrete spectral data, or from two spectra. Recently, the inverse spectral problem for $L$ was studied with mixed spectral data in [7-9]. For the case $R_{00}(\lambda)=1, R_{01}(\lambda)=-h$ in (1.2) and $R_{10}(\lambda)=1, R_{11}(\lambda)=H$ in (1.3), the operator $U\left(q, U_{0}, U_{1}\right)$ turns to a classical Sturm-Liouville problem $L(q, h, H)$. Inverse spectral problems and inverse nodal problems of $L(q, h, H)$ have been well studied, the readers can refer to [2,10-21] and the references therein.

The aim of this article is to investigate the inverse spectral and nodal problems for the BVP $L$. We show that the result on the Weyl $m$-function for $L$ also holds by an alternative approach, which is a generalization of the result for the classical Sturm-Liouville operator in [16]. Moreover, the authors show that the operator $L\left(q, U_{0}, U_{1}\right)$ can be uniquely determined up to constant translation by the twin-dense subset in the interior interval.

\section{Preliminaries}

Let $u_{-}(x, \lambda)$ and $u_{+}(x, \lambda)$ be solutions of equation (1.1) with initial conditions

$$
\begin{array}{ll}
u_{-}(0, \lambda)=R_{01}(\lambda), & u_{-}^{\prime}(0, \lambda)=-R_{00}(\lambda), \\
u_{+}(1, \lambda)=R_{11}(\lambda), & u_{+}^{\prime}(1, \lambda)=-R_{10}(\lambda) .
\end{array}
$$

Denote $\lambda=\rho^{2}, \tau=|\operatorname{Im} \rho|$, for sufficiently large $|\lambda|$, we have

$$
\begin{aligned}
& u_{-}(x, \lambda)=\lambda^{r_{0}}\left(\cos \rho x+O\left(\frac{e^{\tau x}}{\rho}\right)\right), \\
& u_{-}^{\prime}(x, \lambda)=\lambda^{r_{0}}\left(-\rho \sin \rho x+O\left(e^{\tau x}\right)\right), \\
& u_{+}(x, \lambda)=\lambda^{r_{1}}\left(\cos \rho(1-x)+O\left(\frac{e^{\tau(1-x)}}{\rho}\right)\right), \\
& u_{+}^{\prime}(x, \lambda)=\lambda^{r_{1}}\left(\rho \sin \rho(1-x)+O\left(e^{\tau(1-x)}\right)\right) .
\end{aligned}
$$

Denote

$$
\Delta(\lambda):=\left[u_{+}, u_{-}\right](x, \lambda)
$$

where $[y, z](x):=y(x) z^{\prime}(x)-y^{\prime}(x) z(x)$ is the Wronskian of $y$ and $z$. Then

$$
\Delta(\lambda)=-R_{01}(\lambda) u_{+}^{\prime}(0, \lambda)-R_{00}(\lambda) u_{+}(0, \lambda)=-U_{0}\left(u_{+}\right)=U_{1}\left(u_{-}\right)
$$

which is called the characteristic function of $L$ (see [4]). By virtue of (2.1), (2.2), and (2.5), we have

$$
\Delta(\lambda)=\lambda^{r_{0}+r_{1}}\left(-\rho \sin \rho+\omega \cos \rho+o\left(e^{\tau}\right)\right) .
$$

Define the Weyl $m$-function $m_{ \pm}(x, \lambda)$ by

$$
m_{-}(x, \lambda)=-\frac{u_{-}^{\prime}(x, \lambda)}{u_{-}(x, \lambda)}, \quad m_{+}(x, \lambda)=\frac{u_{+}^{\prime}(x, \lambda)}{u_{+}(x, \lambda)}
$$


then

$$
\begin{aligned}
& m_{-}(x, \lambda)=i \rho+o(1) \quad\left(\text { resp. } m_{+}(x, \lambda)=i \rho+o(1)\right) \\
& \frac{1}{m_{-}(x, \lambda)}=-\frac{i}{\rho}+o\left(\frac{1}{\rho^{2}}\right) \quad\left(\text { resp. } \frac{1}{m_{+}(x, \lambda)}=-\frac{i}{\rho}+o\left(\frac{1}{\rho^{2}}\right)\right)
\end{aligned}
$$

uniformly in $x \in[\delta, 1]$ (resp. $x \in[0,1-\delta]$ ) for $|\lambda| \rightarrow \infty$ in any sector $\varepsilon<\arg (\lambda)<\pi-\varepsilon$ for $\varepsilon>0$, where $\delta \in(0,1)$.

Denote the spectrum $\sigma(L):=\left\{\lambda_{n}\right\}_{n=0}^{\infty}$ of $L, \sigma(L)$ consisting of the zeros (counting with multiplicities) of the entire function $\Delta(\lambda)$. For $n$ sufficiently large, $\lambda_{n}$ are real and simple and satisfy the asymptotic formulae (see [4])

$$
\rho_{n}:=\sqrt{\lambda_{n}}=\left(n-r_{0}-r_{1}\right) \pi+\frac{\omega}{n \pi}+\frac{\kappa_{n}}{n}, \quad\left\{\kappa_{n}\right\} \in l^{2},
$$

where

$$
\omega=\frac{1}{2} \int_{0}^{1} q(t) d t-a_{000}+a_{100}
$$

\section{Inverse spectral problems}

For convenience, let $\widetilde{L}=L\left(\widetilde{q}, \widetilde{U}_{0}, \widetilde{U}_{1}\right)$, where $L\left(\widetilde{q}, \widetilde{U}_{0}, \widetilde{U}_{1}\right)$ is the operator of the same form as $L$. If a certain symbol $\gamma$ denotes an object related to $L$, then the corresponding symbol $\tilde{\gamma}$ denotes the analogous object related to $\widetilde{L}$ and $\widehat{\gamma}=\gamma-\tilde{\gamma}$.

The following two theorems on the Weyl $m$-function of the BVP $L$ are derived from [4], and they are generalizations of the analogical results for the classical Sturm-Liouville in [16].

Theorem 3.1 Let $m_{-}\left(a_{0}, \lambda\right)$ be the Weyl m-function of the BVP L. Then $m_{-}\left(a_{0}, \lambda\right)$ can uniquely determine functions $R_{0 k}(\lambda)$ for $k=0$ and 1 as well as $q$ (a.e.) on the interval $\left[0, a_{0}\right]$, $0<a_{0} \leq 1$.

Proof Denote by $L_{D}$ the boundary value problem (1.1), (1.2) together with $\Delta_{D}(\lambda):=$ $u_{-}\left(a_{0}, \lambda\right)=0$ and $\left\{\mu_{a_{0}, n}\right\}_{n=1}^{\infty}$, the zeros (counting with multiplicities) of the entire function $\Delta_{D}(\lambda)$ (see [10]). Then $\mu_{a_{0}, n}$ is real and simple for sufficiently large $n$ and

$$
u_{-}\left(a_{0}, \lambda\right)=\lambda^{r_{0}}\left(\cos \left(a_{0} \rho\right)+\omega_{1} \frac{\sin \left(a_{0} \rho\right)}{\rho}+o\left(\frac{\mathrm{e}^{a_{0} \tau}}{\rho}\right)\right) \text { for }|\rho| \gg 1 \text {, }
$$

where $\omega_{1}=\frac{1}{2} \int_{0}^{a_{0}} q(x) d x-a_{000}$. Thus we have

$$
\sqrt{\mu_{a_{0}, n}}=\frac{\pi}{a_{0}}\left(n-r_{0}+\frac{1}{2}+\frac{\omega_{1}}{n \pi}+o\left(\frac{1}{n}\right)\right) \text { for } n \gg 1 \text {. }
$$

By virtue of Hadamard's factorization theorem,

$$
u_{-}\left(a_{0}, \lambda\right)=C_{a_{0}, 0} \lambda^{m_{0}} \prod_{\mu a_{0}, n \neq 0}\left(1-\frac{\lambda}{\mu_{a_{0}, n}}\right),
$$


where $C_{a_{0}, 0}$ is a constant and $m_{0} \geq 0$. Let $G_{\delta_{0}}:=\left\{\lambda:\left|\rho-\frac{\pi}{a_{0}}\left(k-r_{0}+\frac{1}{2}\right)\right|>\delta_{0}, k \in \mathbb{Z}\right\}$, where $\delta_{0}$ is sufficiently small, then there exists a constant $C_{a_{0}, \delta_{0}}$ (see $\left.[10,11]\right)$ such that

$$
\left|\Delta_{D}(\lambda)\right| \geq C_{a_{0}, \delta_{0}}|\rho|^{2 r_{0}} \mathrm{e}^{a_{0} \tau}, \quad \forall \lambda \in G_{\delta_{0}} \text { and }|\lambda| \gg 1 .
$$

Similarly, denote by $L_{N}$ the boundary value problem (1.1), (1.2) together with $\Delta_{N}(\lambda):=$ $u_{-}^{\prime}\left(a_{0}, \lambda\right)=0$ and $\lambda_{a_{0}, n}$, the zeros (counting with multiplicities) of the entire function $\Delta_{N}(\lambda)$. Then $\left\{\mu_{a_{0}, n}\right\}_{n=1}^{\infty}$ are real and simple for sufficiently large $n$ and

$$
u_{-}^{\prime}\left(a_{0}, \lambda\right)=\lambda^{r_{0}}\left(-\rho \sin \left(a_{0} \rho\right)+c_{0} \cos \left(a_{0} \rho\right)+o\left(\mathrm{e}^{a_{0} \tau}\right)\right)
$$

where $c_{0}=2 r_{0}+\omega_{1}$. Therefore we have

$$
\sqrt{\lambda_{a_{0}, n}}=\frac{\pi}{a_{0}}\left(n-r_{0}+\frac{\omega_{1}}{n \pi}+o\left(\frac{1}{n}\right)\right)
$$

Let $G_{\delta_{1}}:=\left\{\lambda:\left|\rho-\frac{\left(k-r_{0}\right) \pi}{a_{0}}\right|>\delta_{1}, k \in \mathbb{Z}\right\}$, where $\delta_{1}$ is sufficiently small, then there exists a constant $C_{a_{0}, \delta_{1}}$ such that, for sufficiently large $|\lambda|$,

$$
\left|\Delta_{N}(\lambda)\right| \geq C_{a_{0}, \delta_{1}}|\rho|^{2 r_{0}+1} \mathrm{e}^{a_{0} \tau}, \quad \forall \lambda \in G_{\delta_{1}} .
$$

Thus we have

$$
u_{-}^{\prime}\left(a_{0}, \lambda\right)=C_{a_{0}, 1} \lambda^{m_{1}} \prod_{\lambda_{a_{0}, n \neq 0}}\left(1-\frac{\lambda}{\lambda_{a_{0}, n}}\right),
$$

where $m_{1} \geq 0, C_{a_{0}, 1}$ is a constant. Under the assumption $m_{-}\left(a_{0}, \lambda\right)=\widetilde{m}_{-}\left(a_{0}, \lambda\right)$, we obtain

$$
\frac{u_{-}^{\prime}\left(a_{0}, \lambda\right)}{u_{-}\left(a_{0}, \lambda\right)}=\frac{\widetilde{u}_{-}^{\prime}\left(a_{0}, \lambda\right)}{\widetilde{u}_{-}\left(a_{0}, \lambda\right)}
$$

Since $u_{-}\left(a_{0}, \lambda\right)$ and $u_{-}^{\prime}\left(a_{0}, \lambda\right)$ (resp. $\widetilde{u}_{-}\left(a_{0}, \lambda\right)$ and $\left.\widetilde{u}_{-}^{\prime}\left(a_{0}, \lambda\right)\right)$ have no common zeros, $\frac{\widetilde{u}_{-}\left(a_{0}, \lambda\right)}{u_{-}\left(a_{0}, \lambda\right)}$ and $\frac{\widetilde{u}_{-}^{\prime}\left(a_{0}, \lambda\right)}{u_{-}^{\prime}\left(a_{0}, \lambda\right)}$ are two entire functions in $\lambda$ and

$$
\frac{\tilde{u}_{-}\left(a_{0}, \lambda\right)}{u_{-}\left(a_{0}, \lambda\right)}=\frac{\tilde{u}_{-}^{\prime}\left(a_{0}, \lambda\right)}{u_{-}^{\prime}\left(a_{0}, \lambda\right)} .
$$

By virtue of (3.8) together with (2.1), (2.10), (2.2), and (3.4), this yields

$$
\begin{aligned}
& \left|\frac{\widetilde{u}_{-}\left(a_{0}, \lambda\right)}{u_{-}\left(a_{0}, \lambda\right)}\right| \leq O(1), \quad \forall \lambda \in G_{\delta_{0}}, \\
& \left|\frac{\widetilde{u}_{-}^{\prime}\left(a_{0}, \lambda\right)}{u_{-}^{\prime}\left(a_{0}, \lambda\right)}\right| \leq O(1), \quad \forall \lambda \in G_{\delta_{1}} .
\end{aligned}
$$

Using the maximum modulus principle and Liouville's theorem, we have

$$
\left\{\begin{array}{l}
\frac{\widetilde{u}_{-}\left(a_{0}, \lambda\right)}{u_{-}\left(a_{0}, \lambda\right)} \equiv c, \quad \forall \lambda \in \mathbb{C}, \\
\frac{\widetilde{u}_{-}^{\prime}\left(a_{0}, \lambda\right)}{u_{-}^{\prime}\left(a_{0}, \lambda\right)} \equiv c, \quad \forall \lambda \in \mathbb{C} .
\end{array}\right.
$$


Letting $\rho=i y \rightarrow \infty$, then either the first formula in (3.9) together with (2.3) or the second formula in (3.9) together with (2.4) implies

$$
c= \pm 1
$$

By virtue of (2.1), (2.2), and (3.10), we have

$$
c=1
$$

Therefore,

$$
u_{-}\left(a_{0}, \lambda\right)=\tilde{u}_{-}\left(a_{0}, \lambda\right), \quad \text { and } \quad u_{-}^{\prime}\left(a_{0}, \lambda\right)=\widetilde{u}_{-}^{\prime}\left(a_{0}, \lambda\right) \quad \forall \lambda \in \mathbb{C} \text {. }
$$

This implies

$$
u_{-}(x, \lambda)=\widetilde{u}_{-}(x, \lambda), \quad \forall x \in\left[0, a_{0}\right]
$$

Thus (3.11) shows

$$
q(x) \stackrel{\text { a.e. }}{=} \widetilde{q}(x) \quad \text { on }\left[0, a_{0}\right] \quad \text { and } \quad R_{0 k}(\lambda)=\widetilde{R}_{0 k}(\lambda), \quad k=0,1 .
$$

Therefore the proof of Theorem 3.1 is completed.

Analogously, we prove the following theorem on the Weyl $m$-function $m_{+}\left(a_{0}, \lambda\right)$.

Theorem 3.2 Let $m_{+}\left(b_{0}, \lambda\right)$ be the Weyl m-function of the BVP L. Then $m_{+}\left(b_{0}, \lambda\right)$ can uniquely determine functions $R_{1 k}(\lambda)$ for $k=0,1$ as well as $q$ (a.e.) on the interval $\left[b_{0}, 1\right]$, $0 \leq b_{0}<1$.

\section{Inverse nodal problems}

By virtue of Lemma 3.1 in [22], we see that, for $n \gg 1$, the eigenfunction $u_{-}\left(x, \lambda_{n}\right)$ has exactly $n-r_{0}-r_{1}$ zeros $0<x_{n}^{1}<x_{n}^{2}<\cdots<x_{n}^{j}<\cdots<x_{n}^{n-r_{0}-r_{1}}<1$ inside the interval $(0,1)$ and satisfy the following asymptotic formula:

$$
x_{n}^{j}=\frac{j-\frac{1}{2}}{n-r_{0}-r_{1}}-\frac{\left(j-\frac{1}{2}\right) \omega}{n\left(n-r_{0}-r_{1}\right)^{2} \pi^{2}}+\frac{\frac{1}{2} \int_{0}^{x_{n}^{j}} q(t) d t-a_{000}}{\left(n-r_{0}-r_{1}\right)^{2} \pi^{2}}+O\left(\frac{1}{n^{3}}\right)
$$

for $0<j<n-r_{0}-r_{1}$, where $w$ is as that in (2.10). Denote $x_{n}^{0}=0, x_{n}^{n-r_{0}-r_{1}+1}=1$. Note that $\sigma(L)$ might contain non-real eigenvalues, hence we write

$$
\sigma(L)=\sigma_{R}(L) \cup \sigma_{C}(L),
$$

where $\sigma_{R}(L)$ consists of real eigenvalues of $L$. Denote by $X$ the collection of all zeros of all eigenfunctions of $L$. Let $B=\left\{n_{k}\right\}_{k=1}^{\infty}$ be a strictly increasing sequence in $\mathbb{N}$, where $\lambda_{n_{k}} \in$ $\sigma_{R}(L)$. For $0 \leq a<b \leq 1$, we call the subset $W_{B}([a, b])$ of $X \cap[a, b]$ an interior twin-dense nodal subset on the interval $[a, b]$ if the following conditions hold:

(1) For all $n_{k} \in B$, there exists some $j_{k}$ such that $x_{n_{k}}^{j_{k}} \in W_{B}([a, b])$. 
(2) The nodal subset $W_{B}([a, b])$ is twin on the interval $[a, b]$, i.e., if $x_{n_{k}}^{j_{k}} \in W_{B}([a, b])$, then $x_{n_{k}}^{j_{k}+1} \in W_{B}([a, b])$ or $x_{n_{k}}^{j_{k}-1} \in W_{B}([a, b])$.

(3) The nodal subset $W_{B}([a, b])$ is dense on the set $[a, b]$, i.e., $\bar{W}_{B}([a, b])=[a, b]$, where $\bar{W}_{B}([a, b])$ denotes the closure of $\bar{W}_{B}([a, b])$.

The following Lemma 4.1 is necessary for us to prove our main results.

Lemma 4.1 (Theorem $3.2[8])$ If $W_{B}([a, b])=\widetilde{W}_{\widetilde{B}}([a, b])$, then

$$
\left\{\begin{array}{l}
r_{0}+r_{1}=\widetilde{r}_{0}+\widetilde{r}_{1}, \\
q(x)-\tilde{q}(x) \stackrel{\text { a.e. }}{=} 2 \widehat{\omega} \quad \text { on }[a, b], \\
\lambda_{n_{k}}-\tilde{\lambda}_{\widetilde{n}_{k}}=2 \widehat{\omega}, \quad \forall n_{k} \in B, \\
n_{k}=\widetilde{n}_{k} \quad \text { except for a finite number of } k .
\end{array}\right.
$$

Let $S_{B}=\left\{\lambda_{n_{k}}: n_{k} \in B\right\}$. For any sequence $S=\left\{x_{n}\right\}_{n=0}^{\infty}$ of positive real numbers, we define

$$
N_{S}(t)=\#\left\{n \in \mathbb{N} \cup\{0\}: x_{n}<t\right\} .
$$

The following theorem is our main result which concerns the unique determination of the operator from a twin-dense nodal subset and a partial spectrum.

Theorem 4.2 $0<a<\frac{1}{2}<b<1$. Suppose $W_{B}([a, b])=\widetilde{W}_{\widetilde{B}}([a, b])$ and

$$
N_{S_{B}}(t) \geq 2 a_{1} N_{\sigma_{R}(L)}(t)+2\left(1-a_{1}\right)\left(r_{0}+r_{1}+\frac{1}{2}\right)+2 a_{1} k_{0}-1, \quad t \gg 1
$$

for $a_{1}=a$ and $1-b$, where $k_{0}$ is the number of elements in $\sigma_{c}(L)$. Then

$$
\widetilde{q}(x) \stackrel{\text { a.e. }}{=} q(x)-c \text { on }[0,1] \text { and } \widetilde{R}_{\xi k}(\lambda)=R_{\xi k}(\lambda+c) \quad \text { for } \xi, k=0,1 \text {, }
$$

and some constant $c$.

Proof From Lemma 4.1, we have $r_{0}+r_{1}=\widetilde{r}_{0}+\widetilde{r}_{1}$, and $r_{0}+r_{1}$ can be reconstructed from (4.1). By virtue of (3.2), one can reconstruct $\omega$ by

$$
\begin{aligned}
& \lim _{k \rightarrow \infty} n_{k}\left(\sqrt{\lambda_{n_{k}}}-\left(n_{k}-r_{0}-r_{1}\right) \pi\right)=\lim _{k \rightarrow \infty}\left(\omega+\kappa_{n_{k}}\right)=\omega, \\
& \lim _{k \rightarrow \infty} n_{k}\left(\sqrt{\tilde{\lambda}_{n_{k}}}-\left(n_{k}-r_{0}-r_{1}\right) \pi\right)=\lim _{k \rightarrow \infty}\left(\omega+\kappa_{n_{k}}\right)=\widetilde{\omega},
\end{aligned}
$$

and

$$
\left\{\begin{array}{l}
q(x)-\widetilde{q}(x) \stackrel{\text { a.e. }}{=} 2 \widehat{w} \quad \text { on }[a, b], \\
\lambda_{n_{k}}-\tilde{\lambda}_{\widetilde{n}_{k}}=2 \widehat{w}, \quad \forall n_{k} \in B .
\end{array}\right.
$$

Denote

$$
F\left(x, u_{-}, \widetilde{u}_{-}, \lambda\right)=u_{-}(x, \lambda) \widetilde{u}_{-}^{\prime}(x, \lambda-2 \widehat{w})-u_{-}^{\prime}(x, \lambda) \widetilde{u}_{-}(x, \lambda-2 \widehat{w}) .
$$


Then

$$
\begin{aligned}
F\left(a, u_{-}, \tilde{u}_{-}, \lambda\right) & =u_{-}(a, \lambda) \widetilde{u}_{-}^{\prime}(a, \lambda)-u_{-}^{\prime}(a, \lambda) \widetilde{u}_{-}(a, \lambda) \\
& =u_{-}^{\prime}(a, \lambda) \widetilde{u}_{-}^{\prime}(a, \lambda-2 \widehat{w})\left(m_{-}^{-1}(a, \lambda)-\widetilde{m}_{-}^{-1}(a, \lambda-2 \widehat{w})\right) .
\end{aligned}
$$

From (2.2), (2.8), and (4.11), we obtain

$$
\left|F\left(a, u_{-}, \widetilde{u}_{-}, i x\right)\right|=o\left(|y|^{r_{0}+\tilde{r}_{0}} \mathrm{e}^{|2 \operatorname{Im} \sqrt{i x}| a}\right) \quad \text { for }|x| \gg 1, x \in \mathbb{R} .
$$

Moreover, we can choose $\left\{x_{n_{k}}^{j_{n_{k}}}\right\} \in W_{B}([a, b])$ and apply Green's formula to obtain

$$
\begin{aligned}
& \left.\left(\widetilde{u}\left(x, \widetilde{\lambda}_{n_{k}}\right) u^{\prime}\left(x, \lambda_{n_{k}}\right)-\widetilde{u}^{\prime}\left(x, \tilde{\lambda}_{n_{k}}\right) u\left(x, \lambda_{n_{k}}\right)\right)\right|_{a} ^{j_{n_{k}}^{j_{n_{k}}}} \\
& =-\int_{a}^{x_{n_{k}}}\left[\lambda_{n_{k}}-\tilde{\lambda}_{n_{k}}-(q(x)-\widetilde{q}(x))\right] u\left(x, \lambda_{n_{k}}\right) \widetilde{u}\left(x, \widetilde{\lambda}_{n_{k}}\right) d x=0,
\end{aligned}
$$

i.e.,

$F\left(a, u_{-}, \tilde{u}_{-}, \lambda_{n_{k}}\right)=0 \quad$ for all $n_{k} \in B$.

Define the functions

$$
\begin{aligned}
& G_{B}(\lambda)=\prod_{n_{k} \in B}\left(1-\frac{\lambda}{\lambda_{n_{k}}}\right), \\
& \Delta_{R}(\lambda)=\prod_{\lambda_{n} \in \sigma_{R}(L)}\left(1-\frac{\lambda}{\lambda_{n}}\right),
\end{aligned}
$$

and

$$
\Delta_{C}(\lambda)=\prod_{\lambda_{n} \in \sigma_{C}(L)}\left(1-\frac{\lambda}{\lambda_{n}}\right)
$$

Then we know $\Delta_{C}(\lambda)$ is a polynomial of degree $k_{0}$ and

$$
\Delta(\lambda)=K \Delta_{C}(\lambda) \Delta_{R}(\lambda)
$$

for some constant $K$.

Next, we shall use the technique in Appendix B of [15] to get an estimate of $\left|G_{S_{B}}(i x)\right|$. Without loss of generality, we may assume $\lambda>1$ for $\lambda \in \sigma_{R}(L)$ (it can be done by a shift of the parameter $\lambda$ in $L)$. Then $N_{S_{B}(t)}=N_{\sigma_{R}(L)}(t)=0$ for $t \leq 1$, and

$$
\begin{aligned}
\ln |G(i x)| & =\sum_{k=1}^{\infty} \frac{1}{2} \ln \left(1+\frac{x^{2}}{\lambda_{n_{k}}^{2}}\right)=\frac{1}{2} \int_{1}^{\infty} \ln \left(1+\frac{x^{2}}{t^{2}}\right) d N_{S_{B}}(t) \\
& =\frac{1}{2}\left(\left.\ln \left(1+\frac{x^{2}}{t^{2}}\right) N_{S_{B}}(t)\right|_{0} ^{\infty}-\int_{1}^{\infty} N_{S_{B}}(t) \frac{d}{d t}\left(\ln \left(1+\frac{x^{2}}{t^{2}}\right)\right) d t\right) \\
& =-\frac{1}{2} \int_{1}^{\infty} N_{S_{B}}(t) \frac{d}{d t}\left(\ln \left(1+\frac{x^{2}}{t^{2}}\right)\right) d t=\int_{1}^{\infty}\left(\frac{x^{2}}{t^{3}+t x^{2}}\right) N_{S_{B}}(t) d t
\end{aligned}
$$


Hence

$$
\ln \frac{|G(i x)|}{\left|\Delta_{R}(i x)\right|^{2 a}}=\int_{1}^{\infty}\left(\frac{x^{2}}{t^{3}+t x^{2}}\right)\left(N_{S_{B}}(t)-2 a N_{\sigma_{R}(L)}\right) d t .
$$

By (4.6), we know that there exist a $t_{0}$ and a positive number $K$ so that

$$
\begin{cases}N_{S_{B}}(t)-2 a_{1} N_{\sigma_{R}(L)}(t) \geq-K & \text { for } 1 \leq t \leq t_{0}, \\ N_{S_{B}}(t)-2 a_{1} N_{\sigma_{R}(L)}(t) \geq 2\left(1-a_{1}\right)\left(r_{0}+r_{1}+\frac{1}{2}\right)+2 a_{1} k_{0}-1 & \text { for } t>t_{0}\end{cases}
$$

for $a_{1}=a$ or $1-b$. This leads to

$$
\begin{aligned}
\ln \frac{|G(i x)|}{\left|\Delta_{R}(i x)\right|^{2 a_{1}}} \geq & \int_{1}^{t_{0}}[(K+L) / 2] \frac{d}{d t}\left(\ln \left(1+\frac{x^{2}}{t^{2}}\right)\right) d t \\
& +L \int_{t_{0}}^{\infty}(-1 / 2) \frac{d}{d t}\left(\ln \left(1+\frac{x^{2}}{t^{2}}\right)\right) d t
\end{aligned}
$$

where $L=\left[2\left(1-a_{1}\right)\left(r_{0}+r_{1}+\frac{1}{2}\right)+2 a_{1} k_{0}-1\right]$. Hence

$$
\begin{aligned}
& |G(i x)|=O\left(\left|\Delta_{R}(i x)\right|^{2 a_{1}}\left|\Delta_{C}(i x)\right|^{2 a_{1}}\left|\Delta_{C}(i x)\right|^{-2 a_{1}}|x|^{\left.2\left(1-a_{1}\right)\right)\left(r_{0}+r_{1}+\frac{1}{2}\right)+2 a_{1} k_{0}-1}\right) \\
& =O\left(|x|^{\left(2 a_{1}\right)\left(r_{0}+r 1+1 / 2\right)} e^{2 a_{1} \mid \operatorname{Im} \sqrt{i x \mid}}|x|^{-2 a_{1} k_{0}}|x|^{2\left(1-a_{1}\right)\left(r_{0}+r_{1}+\frac{1}{2}\right)+2 a_{1} k_{0}-1}\right) \\
& =O\left(|x|^{2\left(r_{0}+r_{1}\right)} e^{2 a_{1}|\operatorname{Im}(\sqrt{i x})|}\right),
\end{aligned}
$$

i.e.,

$$
\left|G_{S_{B}}(i x)\right| \geq c|x|^{2\left(r_{0}+r_{1}\right)} e^{2 a_{1} \operatorname{Im} \sqrt{i}|x|^{\frac{1}{2}}} \quad \text { for }|x| \gg 1
$$

and $a_{1}=a$ and $1-b$, where $c$ is a constant. Therefore

$$
K_{1}(\lambda):=\frac{F(a, u, \tilde{u}, \lambda)}{G(\lambda)}
$$

is an entire function and

$$
K_{1}(i x) \rightarrow 0 \quad \text { as }|x| \rightarrow \infty
$$

In addition, we easily prove that the following formula

$$
\inf _{|\lambda|=R_{k}}\left|G_{S_{B}}(\lambda)\right| \geq c_{0} \exp ^{-C_{0} R_{k}^{\frac{1+\varepsilon_{0}}{2}}}
$$

holds for sufficiently large $R_{k}$, some $0<\varepsilon_{0}<1, c_{0}$ and $C_{0}$ are two positive constants. Thus, we have

$$
\sup _{|\lambda|=R_{k}}\left|K_{1}(\lambda)\right| \leq c_{0} \exp ^{C_{0} R_{k}^{\frac{1+\varepsilon_{0}}{2}}}
$$


for sufficiently large $R_{k} \rightarrow \infty$ as $k \rightarrow+\infty$. By virtue of (4.21) and (4.22) (see [11], Proposition B.6), we obtain

$$
K_{1}(\lambda)=0, \quad \forall \lambda \in \mathbb{C} .
$$

Therefore, we get

$$
F\left(a, u_{-}, \widetilde{u}_{-}, \lambda\right)=0, \quad \forall \lambda \in \mathbb{C} .
$$

This implies

$$
m_{-}(a, \lambda)=\widetilde{m}_{-}(a, \lambda-2 \widehat{w}) .
$$

By Theorem 3.1 together with (4.24), we get

$$
\widetilde{q}(x) \stackrel{\text { a.e. }}{=} q(x)-2 \widehat{w} \quad \text { on }[0, a], \quad \text { and } \quad \widetilde{R}_{0 k}(\lambda)=R_{0 k}(\lambda+2 \widehat{w}), \quad k=0,1 .
$$

Similarly, we can define

$$
H\left(x, u_{+}, \tilde{u}_{+}, \lambda\right)=u_{+}(x, \lambda) \widetilde{u}_{+}^{\prime}(x, \lambda-2 \widehat{w})-u_{+}^{\prime}(x, \lambda) \widetilde{u}_{+}(x, \lambda-2 \widehat{w}) .
$$

Then one can repeat the same arguments as above on $H\left(b, u_{+}, \tilde{u}_{+}, \lambda\right)$ to show

$$
\frac{H\left(b, u_{+}, \tilde{u}_{+}, \lambda\right)}{G_{B}(\lambda)}=0 .
$$

This leads to $H\left(b, u_{+}, \tilde{u}_{+}, \lambda\right)=0$ and

$$
m_{+}(b, \lambda)=\widetilde{m}_{+}(b, \lambda-2 \widehat{w}) .
$$

Hence

$$
\widetilde{q}(x) \stackrel{\text { a.e. }}{=} q(x)-2 \widehat{w} \quad \text { on }[b, 1], \quad \text { and } \quad \widetilde{R}_{1 k}(\lambda)=R_{1 k}(\lambda+2 \widehat{w}), \quad k=0,1 .
$$

(4.9), (4.25), and (4.27) imply

$$
\widetilde{q}(x) \stackrel{\text { a.e. }}{=} q(x)-2 \widehat{w} \quad \text { on }[0,1] \quad \text { and } \quad \widetilde{R}_{\xi k}(\lambda)=R_{\xi k}(\lambda+2 \widehat{w}), \quad \xi, k=0,1 .
$$

This completes the proof of Theorem 4.2.

Corollary 4.3 Under the assumptions of Theorem 4.2, if $\lambda_{n_{k}}=\widetilde{\lambda}_{n_{k}}$ for $n_{k} \gg 1$, then $q(x)=$ $\widetilde{q}(x)$ and $R_{i j}(\lambda)=\widetilde{R}_{i, j}(\lambda)$ for $i, j=0,1$.

Remark The readers might be interested in the inverse nodal problem for a more general equation

$$
\left\{\begin{array}{l}
u^{\prime \prime}+A(\lambda) u=0, \quad x \in(0,1), \\
R_{01}(\lambda) u^{\prime}(0)+R_{00} u(0)=0, \\
R_{11}(\lambda) u^{\prime}(1)+R_{10} u(1)=0,
\end{array}\right.
$$


where $\frac{d^{2}}{d x^{2}}+A(\lambda)$ is an operator on $H^{2}(a, b)$. Some of such problems arise from PDE (please refer to $[23,24]$ for details). Same arguments for Theorem 4.2 seem to work for (4.28) if $A(\lambda)$ is an appropriate operator.

\section{Conclusion}

In this paper, the authors show that a twin-dense subset $W_{B}([a, b]), 0<a<1 / 2<b<1$, can uniquely determine (up to a constant translation on both boundary conditions and potential) the Sturm-Liouville operator associated with boundary conditions polynomially dependent on the spectral parameter. The theorem leads to the same conclusion for classical Sturm-Liouville equation when the coefficient polynomials $R_{i j}(\lambda)$ are all of degree 0 (refer to [25]), but the translation effect on boundary conditions only appears when one of $R_{i j}(\lambda)$ is a non-trivial polynomial.

\section{Acknowledgements}

The authors acknowledge the reviewers for their helpful comments.

\section{Funding}

The third author was partially supported by the Ministry of Science and Technology of Taiwan grant 106-2115-M-032-004-.

\section{Availability of data and materials}

Not applicable.

\section{Competing interests}

The authors declare that they have no competing interests.

\section{Authors' contributions}

The authors contributed equally in this article. They have all read and approved the final manuscript.

\section{Author details}

'Department of Applied Mathematics, Nanjing Forestry University, Nanjing, People's Republic of China. ${ }^{2}$ Department of Mathematics, Tamkang University, New Taipei City, Taiwan.

\section{Publisher's Note}

Springer Nature remains neutral with regard to jurisdictional claims in published maps and institutional affiliations.

Received: 30 November 2017 Accepted: 22 February 2018 Published online: 05 March 2018

References

1. Binding, P.A., Browne, P.J., Seddighi, K.: Sturm-Liouville problems with eigenparameter dependent boundary conditions. Proc. R. Soc. Edinb. 37, 57-72 (1993)

2. Browne, P.J., Sleeman, B.D.: Inverse nodal problem for Sturm-Liouville equation with eigenparameter dependent boundary conditions. Inverse Probl. 12, 377-381 (1996)

3. Chernozhukova, A.Y., Freiling, G.: A uniqueness theorem for inverse spectral problems depending nonlinearly on the spectral parameter. Inverse Probl. Sci. Eng. 17, 777-785 (2009)

4. Freiling, G., Yurko, V.A.: Inverse problems for Sturm-Liouville equations with boundary conditions polynomially dependent on the spectral parameter. Inverse Probl. 26(6), 055003 (2010)

5. Ozkan, A.S., Keskin, B.: Spectral problems for Sturm-Liouville operator with boundary and jump conditions linearly dependent on the eigenparameter. Inverse Probl. Sci. Eng. 20(6), 799-808 (2012)

6. Wang, Y.P.: Inverse problems for discontinuous Sturm-Liouville operators with mixed spectral data. Inverse Probl. Sci. Eng. 23, 1180-1198 (2015)

7. Wang, Y.P.: Inverse problems for a class of Sturm-Liouville operators with the mixed spectral data. Oper. Matrices 11, 89-99 (2017)

8. Wang, Y.P., Lien, K.Y., Shieh, C.T.: Inverse problems for the boundary value problem with the interior nodal subsets. Appl. Anal. 96(7), 1229-1239 (2017)

9. Wang, Y.P., Yurko, V.A.: On the inverse nodal problems for discontinuous Sturm-Liouville operators. J. Differ. Equ. 260, 4086-4109 (2016)

10. Buterin, S.A., Shieh, C.T.: Inverse nodal problem for differential pencils. Appl. Math. Lett. 22, 1240-1247 (2009)

11. Cheng, Y.H., Law, C.K., Tsay, J.: Remarks on a new inverse nodal problem. J. Math. Anal. Appl. 248, 145-155 (2000)

12. Chen, X.F., Cheng, Y.H., Law, C.K.: Reconstructing potentials from zeros of one eigenfunction. Trans. Am. Math. Soc. $363,4831-4851$ (2011)

13. Everitt, W.N.: On a property of the m-coefficient of a second-order linear differential equation. J. Lond. Math. Soc. 4, 443-457 (1972) 
14. Freiling, G., Yurko, V.A.: Inverse Sturm-Liouville Problems and Their Applications. Nova Science Publishers, Huntington (2001)

15. Gesztesy, F., Simon, B.: Inverse spectral analysis with partial information on the potential, II. The case of discrete spectrum. Trans. Am. Math. Soc. 352, 2765-2787 (2000)

16. Marchenko, V.A.: Some questions in the theory of one-dimensional linear differential operators of the second order, I. Tr. Mosk. Mat. Obŝ. 1, 327-420 (1952) (Russian); English transl. in Amer. Math. Soc. Transl. 2, 101(1973), 1-104

17. McLaughlin, J.R.: Inverse spectral theory using nodal points as data-a uniqueness result. J. Differ. Equ. 73, 354-362 (1988)

18. Shieh, C.T., Yurko, V.A.: Inverse nodal and inverse spectral problems for discontinuous boundary value problems J. Math. Anal. Appl. 347, 266-272 (2008)

19. Wei, G.S., Xu, H.K.: Inverse spectral problem with partial information given on the potential and norming constants. Trans. Am. Math. Soc. 364, 3265-3288 (2012)

20. Yang, C.F., Yang, X.P.: Inverse nodal problem for the Sturm-Liouville equation with polynomially dependent on the eigenparameter. Inverse Probl. Sci. Eng. 19, 951-961 (2011)

21. Yurko, V.A.: Inverse nodal problems for Sturm-Liouville operators on star-type graphs. J. Inverse III-Posed Probl. 16 715-722 (2008)

22. Yang, X.F.: A new inverse nodal problem. J. Differ. Equ. 169, 633-653 (2001)

23. Shahmurov, R.: Solution of the Dirichlet and Neumann problems for a modified Helmholtz equation in Besov spaces on an annulus. J. Differ. Equ. 249, 526-550 (2010)

24. Shahmurov, R:: On strong solutions of a Robin problem modelling heat conduction in materials with corroded boundary. Nonlinear Anal., Real World Appl. 13, 441-451 (2012)

25. Guo, Y., Wei, G.: Inverse problems: dense nodal subset on an interior subinterval. J. Differ. Equ. 255, 2002-2017 (2013)

\section{Submit your manuscript to a SpringerOpen ${ }^{\circ}$ journal and benefit from:}

- Convenient online submission

- Rigorous peer review

- Open access: articles freely available online

- High visibility within the field

- Retaining the copyright to your article

Submit your next manuscript at springeropen.com 